\title{
Towards An Integrated Blood Pressure Self- Monitoring SolutloN for Stroke/TIA in Ireland: A Mixed Methods Feasibility Study for the TASMIN5S IRL Randomised Controlled Trial
}

\section{Roisin Doogue ( $\nabla$ roisin.doogue@ul.ie )}

University of Limerick https://orcid.org/0000-0002-4539-3982

\section{Peter Hayes}

University of Limerick

\section{Robin Hebert}

University of Limerick

\section{Ali Sheikhi}

University of Limerick

\section{Tanvi Rai}

Oxford University: University of Oxford

\section{Kate Morton}

University of Southampton

\section{Cristian Roman}

Oxford University: University of Oxford

\section{Richard J McManus}

Oxford University: University of Oxford

\section{Liam G Glynn}

University of Limerick

\section{Research}

Keywords: blood pressure, self-monitoring, stroke, feasibility studies, randomised controlled trial, qualitative research.

Posted Date: January 31st, 2022

DOI: https://doi.org/10.21203/rs.3.rs-1263447/v1

License: (9) This work is licensed under a Creative Commons Attribution 4.0 International License. Read Full License 


\section{Abstract \\ Background}

Optimising Blood Pressure (BP) control is one of the most important modifiable risk factors in preventing subsequent stroke where the risk increases by one-third for every $10 \mathrm{mmHg}$ rise in systolic BP. The aim of this study was to evaluate the feasibility and effects of blood pressure self-monitoring with planned medication titration, in patients with a previous stroke or transient ischaemic attack (TIA).

\section{Methods}

Patients with a history of stroke/TIA and sub-optimal BP control, were invited to take part in a mixed methods feasibility study for a randomised controlled trial. Those meeting the inclusion criteria with systolic $\mathrm{BP}>130 \mathrm{mmHg}$, were randomised to a self-monitoring intervention group or usual care group. The intervention involved self-monitoring BP twice a day for 3 days within a 7-day period, every month, following text message reminders. Treatment escalation, based on a pre-agreed plan by the General Practitioner (GP) and patient, was initiated according to the results of these readings. Semi-structured interviews were carried out with patients and clinicians, and analysed thematically.

\section{Results}

Of those identified, 47\% (32/68) attended for assessment. Of those assessed, 15 were eligible for recruitment and were consented and randomised to the intervention or control group on a 2:1 basis. Of those randomised, 93\% (14/15) completed the study and there were no adverse events. Systolic BP was lower in the intervention group at 3 months. Participants found the intervention acceptable and easy to use. GPs found it easy to incorporate into their practice activity without increasing workload.

\section{Conclusions}

TASMIN5S, an integrated blood pressure self-monitoring intervention in patients with a previous stroke/ TIA is feasible and safe to deliver in primary care. A pre-agreed three step medication titration plan was easily implemented, increased patient involvement in their care, and had no adverse effects. This feasibility study provides important information to inform a definitive trial to determine the potential effectiveness of the intervention in patients post stroke or TIA.

\section{Trial Registration:}

ISRCTN57946500, registered on the 12/08/2019. https://doi.org/10.1186/ISRCTN57946500 


\section{Key Messages Regarding Feasibility}

1) What uncertainties existed regarding feasibility prior to this study?

TASMIN5S is amultidimensional intervention designed to help lower blood pressure in patients who have had a previous stroke/TIA. Patients are supported to take and record their own blood pressure, with instant feedback of results.A monthly report is sent to their GP. The GP manages the results based on a pre-agreed treatment plan. Prior to testing the effectiveness of this multi-component intervention we wanted to explore the feasibility and acceptability of implementing this intervention with both patients and GPs, test the randomisation and data collection methods and get feedback from patients and GPs that would inform necessary changes to the design for a full randomised controlled trial (RCT).

2) What are the key feasibility findings?

Patients found the intervention acceptable and easy to use. The majority $(>90 \%)$ completed the 3-month trial and indicated willingness to continue with the intervention. The electronic messaging and feedback system worked without any failures. Valuable information on invitation and recruitment processes was obtained. GPs found the intervention acceptable and easy to implement without adding to their workload. More efficient methods of receiving results in to the practice were identified for further trials.

3) What are the implications of the feasibility findings for the design of the main study?

The findings from this study provided information on the recruitment process and the importance of GP support, and a better understanding of the number of practices that will need to be recruited for a full trial. Many of the volunteers when assessed, were excluded as their BP was well controlled. This is important information for recruitment planning for a full trial. We identified improvements to the results delivery system which will make the intervention more efficient for the GP. All participants in this trial preferred the option of free text messaging to record their BP results. However, having the options of an App and website will continue to be available for patients who might prefer these options in the full trial.

\section{Background}

Stroke is one of the leading causes of death and disability worldwide. Despite improvements in the prevention and management of stroke, the incidence of stroke continues to grow especially with an aging population (1). The economic burden of stroke in Europe is significant, increasing from $€ 45$ billion per year in 2015 (2), to more recent estimated costs of $€ 60$ billion per year (3). The individual effects of stroke are even more significant with many people leaving hospital after a stroke suffering from limb impairment or aphasia (impacting the ability to read, write or understand language), often resulting in devastating personal, social or economic consequences.

We acknowledge that primary prevention of stroke/ TIA (hereafter referred to as stroke) must be our first aim, in order to prevent these debilitating consequences. However, in those who have had a previous 
stroke it is vital that we focus on risk factor modification to prevent further events. Subsequent stroke has an even higher incidence of disability and death, with cumulative risks of further stroke increasing from $3 \%$ after 1 month to $40 \%$ ten years after first stroke (4). Secondary prevention has the potential to reduce stroke reoccurrence by up to $80 \%$. Measures include smoking cessation, reducing salt intake, moderate alcohol intake, achieving a healthy BMI, increased exercise, dietary modifications with increased fruit and vegetable intake and controlling blood pressure to a target of $<130 / 80 \mathrm{mmHg}$ if tolerated (5). Of these, high blood pressure has been shown to be one of the most important modifiable risk factors in the secondary prevention of stroke, with evidence suggesting that lowering systolic BP by $10 \mathrm{mmHg}$ can reduce the incidence of stroke by one third and that further reduction in BP where tolerated, can have an even more beneficial effect, regardless of the blood pressure level before treatment (6).

Controlling blood pressure in this complex group of patients can be challenging. A survey of secondary prevention of stroke in Europe has shown that satisfactory levels of blood pressure control was found in less than $60 \%$ of countries (7). A recent cross-sectional survey in Irish General Practice (8), demonstrated that one third of patients did not have their blood pressure adequately controlled. The reasons for suboptimal BP control are multi-faceted. These include patient factors where adherence may be an issue ( 9 , $10)$, physician factors, which may also include therapeutic inertia (10), lifestyle issues and treatment resistant hypertension (11).

Developing novel strategies to optimise BP control in this group of patients could have potential benefits in reducing the incidence of further stroke. GP supervised self-monitoring and self-management of blood pressure has been shown to be effective in improving blood pressure in primary care $(12,13)$. Due to the often-debilitating outcomes in patients following stroke, it is important to investigate if a self-monitoring intervention can be used by this patient group, and if they are effective.

\section{Aims}

This study aimed to evaluate the feasibility, acceptability and potential effectiveness of an intervention involving blood pressure self-monitoring, with planned medication titration, among patients with a previous stroke/TIA in Ireland.

\section{Methods}

Trial registration and Ethics Approval

This feasibility study was adapted from the TASMIN5S protocol (ISRCTN57946500), a multi-centre RCT examining the effects of blood pressure self-monitoring in patients with a previous stroke or TIA which took place concurrently in the United Kingdom. Research ethics approval was received from University Hospital Limerick Research Ethics Committee (Ref: 077/19). We have followed CONSORT guidelines for the reporting of feasibility studies (14). 
The study design was a prospective two-group, unmasked feasibility study for a randomised controlled trial. Participants were allocated to the intervention or control group on a 2:1 basis. An internet based randomisation process was used. The trial was carried out in the mid-west of Ireland.

Patient and Public Involvement

Stroke patients and advocates from the Stroke Association (www.stroke.org.uk) were included on the advisory committee overseeing the development of the main intervention. Community groups such as Different Strokes Southampton and the Oxford Aphasia Group were asked for feedback during the intervention development.

The intervention development process involved stroke patients from both Ireland and the UK providing an intervention and material suitable for diverse groups of people from both an Irish and UK health care environment. This helped to identify potential barriers and improve the accessibility of the intervention (15), resulting in patient material that was easy-to-read, aphasia friendly and suitable for people with lower literacy levels.

\section{Participants}

General practices in the mid-west of Ireland who had taken part in a recent cross-sectional survey of stroke and TIA (8) were invited to take part in the feasibility study. All practices used electronic medical records (EMRs) and cared for both public (funded through the Primary Care Reimbursement Service (PCRS) scheme (16) and private (fee-paying) patients. Adults over 18 years, with a history of a previous stroke or TIA, with a recent office blood pressure $>130 \mathrm{mmHg}$ systolic, who met the inclusion criteria (Appendix 1), were identified by their GP from their practice EMRs and invited to participate.

\section{Recruitment}

The study commenced in October 2019. Study information was sent to each participating GP, which included study eligibility (Appendix 1) and exclusion (Appendix 2) criteria, a short online information programme for health care professionals and a copy of all study materials. GPs who were willing to participate in the study were asked to send an invitation letter and short study information leaflet (Appendix 3) to identified patients, outlining the proposed feasibility study and inviting them to take part. The patient information leaflet providing details of the study was in pictorial format to allow better understanding for those with possible aphasia post stroke or those with lower literacy levels (15). Patients were invited to return the reply slip giving their permission to be contacted by the researcher in order to receive further information about the study and make an appointment for study eligibility assessment. Patients who did not want to take part could return the reply slip indicating same and a reason why not. Non-responders were followed up by the GP two-three weeks later by phone, and provided with further information. They were invited to return the reply slip if interested. 
Two practices were recruited prior to the Covid-19 lockdown in Ireland (December, 2019- February 2020). Participants who had given their permission, were contacted by the researcher and invited to attend an assessment appointment in their own GP practice. Following informed consent and assessment, eligible patients with $\mathrm{BP}>130 \mathrm{mmHg}$ (average of second and third reading after sitting for 5 minutes, using the BP-TRU monitor (17)) were randomised to the intervention (self-monitoring) or control (usual care) group using an internet based randomisation key (18).

A further two practices were recruited during the Covid-19 lockdown (August 2020-September 2020) following ethical approval for an amendment to the protocol allowing for the recruitment of patients to the study remotely. Participants were assessed by phone or video consultation after receiving all study materials by post. The amended protocol exactly mirrored the original protocol except all interactions with the patient took place virtually.

Baseline data collected during the assessment included: patient characteristics and demographics, level of education, past medical history including co-morbidities, number of antihypertensive medications currently prescribed, short orientation memory concentration test (19) and blood pressure readings including an assessment of orthostatic hypotension.

\section{Randomisation}

Internet based randomisation with phone backup was used (18). The randomisation key was held by an independent researcher not involved in the recruitment or the assessment of participants. When a participant was eligible for recruitment, the independent researcher was contacted by text message and the allocation group was advised. As the main objective in this study was to explore feasibility, randomisation was on a 2:1 intervention to control basis.

Intervention

The TASMIN5S intervention used a free-text telemonitoring platform called BP: Together (established during the intervention development phase (15)), which was set up to allow the sending and receiving of free text messages to mobile phones. Results of the patients' home BP readings were automatically collated from the digital platform and sent to each patient's individual GP by email.

Participating GPs completed a 30-minute online training programme before the study commenced. It outlined the objectives of the study, provided a rationale for lowering stroke patients' blood pressure to the intervention targets ( $<125 \mathrm{mmHg}$ systolic on home readings), gave practical information on how they would receive the patient's home BP readings, provided the GP with a review of current guidelines for BP control in Stroke or TIA $(11,20)$ and provided suggestions on BP management strategies including pharmacological intensification (12).

The self-monitoring intervention group received a BP monitor (Omron M10-IT (21)) and were instructed in how to take their own BP and how to send the BP readings using the digital platform of choice: 
1) free text message to the BP: Together platform,

2) via the BP: Together App downloaded to their phone,

3) or via the BP: Together website.

Pre Covid-19, patients received instruction from the research nurse following assessment and randomisation in their GP surgery, with a take-home instruction booklet provided. During the Covid-19 lockdown, patients received the same instruction from the research nurse by phone/ video consultation, with the instruction booklet provided by post with the BP monitor.

Following the baseline assessment, GPs were sent an email with a list of their patients randomised to the intervention (self-monitoring) and control (usual care) groups. Patients in both groups received a medication review by their GP. During this baseline medication review, patients in the intervention group were given an individualised three-step treatment escalation plan. This plan would be enacted by the GP if there was a clinical indication, based on the results of the home BP readings received by email following each monitoring period. The control group continued with usual care for the duration of the feasibility study.

Self-monitoring involved recording BP twice a day for three days within a seven-day period, every month, following text message reminders. Patients sent their BP readings by free-text to the BP: Together digital platform. The monthly average BP was sent to the patient using a traffic light system (Figure 1) and to the patient's GP immediately after each monitoring period. Treatment escalation was initiated by the GP if indicated, according to the three-step medication plan (Appendix 4). The feasibility study lasted three months.

Post-study interviews

All patients randomised to the intervention arm and all participating GPs were invited to take part in a post-study interview. We developed two semi-structured interview guides - one for patients and one for GPs - in order to obtain their respective feedback about the intervention. The interview guides were developed using a planned approach which identified prerequisites and current knowledge, to encourage patients and the GPs to provide feedback on the intervention (22).

\section{Outcomes}

The outcomes of interest for this study were defined as:

1. the number of GP practices willing to participate in the study and the retention rate,

2. feasibility of recruitment and retention of patients,

3. allocation process and randomisation,

4. patient willingness to adhere to the intervention and to continue with the programme, and the likelihood of recommending the programme to other stroke patients, 
5. the effects of the intervention on GP practice time and workload, and

6 . the number of adverse events recorded during the study.

Sample Size

As this was a feasibility study, there were no formal power calculations before commencing the study (23).

\section{Data Analysis}

Quantitative data analysis was carried out using SPSS (24). Descriptive statistics were used to compare the participants' baseline characteristics. Continuous variables are reported as mean (+/-Standard Deviation (SD)) and categorical variables are presented as count (percentage). Formal tests of statistical significance were not carried out, as this study was not conducted or powered to detect effects on BP.

Thematic analysis was used to explore the emerging themes from the post-trial interviews with both patients and participating GPs. The five stages of the Framework Process were followed in the examination of the qualitative data which included familiarisation, thematic framework identification, indexing, charting, mapping and interpretation (25). Coding was partially conducted with another researcher from a different professional background for inter-coder reliability (26). To heighten reflexivity, three members of the research team (a research nurse and two general practitioners) reviewed all the data and contributed to the thematic analysis (27). QSR International NVivo 12 software (28) was used to organise and code the transcripts to facilitate the analysis and comparison of relationships between the coded ideas (29).

\section{Results}

Eight out of the ten practices invited to take part in the study indicated willingness to participate when contacted initially. Due to the effects of Covid-19, four of these practices withdrew, stating that they did not have the capacity to participate due to the increased workload associated with the Covid-19 pandemic but would have taken part otherwise. In total, four of the ten general practices invited, were recruited.

The four practices that participated were representative of Irish general practice (30) in size and type, teaching and non-teaching. They consisted of a large urban, small urban, large mixed urban and rural, and small rural practice. The initial assessment for trial eligibility, following a computer search of the practice EMRs, identified 233 patients with a previous stroke or TIA. Of these, there were 95 potential patients who met the inclusion criteria (Appendix 1) and were eligible for invitation to the study. Following review by the GP, 27 patients were excluded for the following reasons: BP self-monitoring not suitable for patients, patients had left practice, patients had a diagnosis of dementia which had not been coded correctly and patient deceased. This resulted in 68 patients eligible for invitation. 
Thirty-seven of the patients identified (54.4\%), agreed to attend for assessment. Of these, 18 (26.5\%) agreed to take part after the mailout and a further 19 (27.9\%) agreed to take part following a call from the GP. During the follow up phone call by the GP to non-responders, practical barriers that were identified included concerns about being able to take their own BP and send the BP results as required. The follow up phone call from the GP reassured patients and doubled participation in the study.

Thirty-two of the 37 volunteers attended baseline assessment. Of these 15 were eligible for randomisation. Seventeen patients were not eligible to take part, 16 had BP controlled below $130 \mathrm{mmHg}$ systolic and one person had a new diagnosis of atrial fibrillation. See Consort diagram for further details (Figure 2). The 15 eligible patients were randomised on a 2:1, intervention (self-monitoring) to control (usual care) basis.

Participant characteristics are described in Table 1. These are presented as total participants, intervention and control groups. Baseline characteristics were similar in both study arms. The mean age of the total group was 70 years. $80 \%$ were male and $53.3 \%$ lived alone. Almost three quarters (73.3\%) of participants had suffered a previous stroke while the remaining quarter $(26.7 \%)$ had a TIA. All levels of education were represented, including those who had a primary education only, to post graduate levels of education. 
Table 1

Baseline Characteristics

\begin{tabular}{|c|c|c|c|}
\hline \multirow[t]{3}{*}{ Participant characteristics } & Intervention & Control & Total \\
\hline & Self-Monitoring & Usual Care & $N=15$ \\
\hline & $N=10$ & $N=5$ & \\
\hline \multicolumn{4}{|l|}{ Demographics } \\
\hline Age, mean (SD) & $69(7.1)$ & $71.2(8.7)$ & $70(7.4)$ \\
\hline Male, n (\%) & $8(80 \%)$ & $4(80 \%)$ & $12(80 \%)$ \\
\hline Lives alone, $\mathrm{n}(\%)$ & $6(60 \%)$ & $2(40 \%)$ & $8(53.3 \%)$ \\
\hline Helped by carer, n (\%) & $3(30 \%)$ & $1(20 \%)$ & $4(26.7 \%)$ \\
\hline Stroke, n (\%) & $7(70 \%)$ & $4(80 \%)$ & $11(73.3 \%)$ \\
\hline TIA, n (\%) & $3(30 \%)$ & $1(20 \%)$ & $4(26.7 \%)$ \\
\hline \multicolumn{4}{|l|}{ Education (Highest level achieved) } \\
\hline Primary education only, n (\%) & $2(20 \%)$ & $1(20 \%)$ & $3(20 \%)$ \\
\hline Secondary school certificate, $\mathrm{n}(\%)$ & $3(30 \%)$ & $2(40 \%)$ & $5(33.3 \%)$ \\
\hline University degree or higher, $\mathrm{n}(\%)$ & $4(40 \%)$ & $2(40 \%)$ & $6(40 \%)$ \\
\hline Diploma/ Other qualification, n (\%) & $1(10 \%)$ & 0 & $1(6.7 \%)$ \\
\hline \multicolumn{4}{|l|}{ Co-Morbidities* } \\
\hline Myocardial Infarction, n (\%) & 0 & $1(20 \%)$ & $1(6.7 \%)$ \\
\hline Angina, n (\%) & 0 & $1(20 \%)$ & $1(6.7 \%)$ \\
\hline CABG/ angioplasty (balloon)/ stent, $\mathrm{n}(\%)$ & $3(30 \%)$ & $1(20 \%)$ & $4(26.7 \%)$ \\
\hline Peripheral Vascular Disease, n (\%) & 0 & 0 & 0 \\
\hline Diabetes- Type 1, n (\%) & 0 & 0 & 0 \\
\hline Diabetes- Type 2, n (\%) & $1(10 \%)$ & $1(20 \%)$ & $2(13.3 \%)$ \\
\hline Heart Failure, n (\%) & 0 & 0 & 0 \\
\hline \multicolumn{4}{|l|}{ Current antihypertensive medication } \\
\hline $0, \mathrm{n}(\%)$ & 0 & 0 & 0 \\
\hline $1, \mathrm{n}(\%)$ & $4(40 \%)$ & $1(20 \%)$ & $5(33.3 \%)$ \\
\hline $2, \mathrm{n}(\%)$ & $6(60 \%)$ & $4(80 \%)$ & $10(66.7 \%)$ \\
\hline Range & $1-2$ & $1-2$ & $1-2$ \\
\hline
\end{tabular}




\begin{tabular}{|llll|}
\hline Participant characteristics & Intervention & Control & Total \\
& Self-Monitoring & Usual Care & $\mathbf{N}=15$ \\
& $\mathbf{N}=\mathbf{1 0}$ & $\mathbf{N}=\mathbf{5}$ & \\
\hline *Two patients had more than one co-morbidity & \\
\hline
\end{tabular}

The mean systolic BP at baseline was $143.7 \mathrm{mmHg}$ in the intervention group and $146.2 \mathrm{mmHg}$ in the control group and the mean diastolic BP was $79.3 \mathrm{mmHg}$ and $78.6 \mathrm{mmHg}$ respectively (mean of second and third reading after sitting for five minutes) (Table 2 ).

The three-month feasibility study was completed by 14 out of the 15 participants. The participants who completed the trial adhered to the protocol of monthly BP measurement and recording for three months, sent their readings to the trial platform and agreed treatment adjustment with their GP. All participants chose to text their blood pressure results rather than using the BP: Together App or website. They found the text messaging simple to use and preferred it as many had concerns over unreliable internet access. Two participants received help from a spouse or carer. One needed help to send the results by text message. The other needed help to take the BP readings and send the results. All participants were given a contact number for the research nurse if they had any questions relating to the procedure for taking their BP, recording the results or texting their results. There were no differences in intervention adherence or trial completion between the group assessed and taught how to use the intervention by the research nurse in surgery versus the group at home.

Blood pressure dropped in both intervention and control groups. The drop in both the systolic and diastolic BP readings was higher in the intervention group than that in the control group. Systolic BP dropped from $143.7 \mathrm{mmHg}$ at baseline to $120.8 \mathrm{mmHg}$ post-trial in the intervention group and from 146.2 $\mathrm{mmHg}$ to $140.5 \mathrm{mmHg}$ in the control group. Diastolic BP dropped from $79.3 \mathrm{mmHg}$ to $69.4 \mathrm{mmHg}$ in the intervention group and from $78.6 \mathrm{mmHg}$ to $73.1 \mathrm{mmHg}$ in the control group. However, these cannot be compared for significance due to the small numbers involved, and the primary aim of this study was to test the feasibility of the intervention (Table 2). 
Table 2

BP readings pre and post feasibility study

Intervention

Self-Monitoring $(n=9)$
Control

Usual Care

$(n=5)$

\section{Baseline BP (mean of 2nd and 3rd reading)}

Systolic BP, mean (SD)

$143.7(9.49)$

$146.2(9.42)$

Diastolic BP, mean (SD)

$79.3(11.37)$

$78.6(15.14)$

\section{Post-trial BPa (mean of 2 nd and 3rd reading)}

Systolic BP, mean (SD)

$120.8(16.35)$

$140.5(3.86)$

Diastolic BP, mean (SD)

$69.4(9.07)$

$73.1(14.97)$

${ }^{a}$ The change in BP between baseline and post-trial cannot be compared for statistical significance due to the small numbers in the feasibility study.

Post-trial interviews

All participants who completed the intervention and all GPs consented to a post pilot trial interview. Following thematic analysis, emerging themes from patient interviews included perceived benefits or negative effects of blood pressure self-monitoring and the prescribed intervention, willingness to continue with the intervention and likelihood of recommending the programme to other survivors of stroke. The main themes emerging from the GP interviews were; the benefits of BP self-monitoring, managing the intervention and incorporating it in to practice systems, and the potential impact on their workload.

Participant views

All nine participants who completed the intervention took part in a structured phone interview. The majority of these found the intervention easy to use, acceptable, and didn't cause any anxieties or concerns but on the contrary appeared to reduce anxiety around blood pressure. Pseudonyms are used in place of participant's names.

I think it's great that you can self-monitor and get a better feeling for your own health.

(Mark, 69 years)

I found it very helpful and I relaxed a bit more about my blood pressure knowing that it was being monitored by the doctor.

(Linda, 71 years) 
However, one participant found it caused her to feel anxious even though she wouldn't normally class herself as being an anxious person. She was very pleased when the three-month pilot was over and stated that she would be reluctant to continue in a full 12-month trial.

...it seemed to be very high (BP) and I don't know. In the end I was kind of dreading it.

(Kathleen, 73 years)

This highlights the importance of ensuring patients can access support if they are feeling anxious, especially if their BP readings are high. Support from her GP or the research team may reduce her anxiety and give her the confidence to continue with the intervention.

Some indicated that it motivated them to adhere to a healthier lifestyle including improved diet and increased exercise.

Because when my blood pressure was up, I was cutting back on a couple of things. I was cutting back on salt and butter and this and that so I think it did help me.

(Jack, 57 years)

Many of the participants stated that it encouraged them to become more involved in their care. They discussed their readings with the GP and the possible need for a medication change.

When we were checking over a few weeks or a month or a two-month period, it was obvious that my medication had to be increased, which helped as well, which might never had happened, you know, if I didn't have it.

(Mark, 69 years)

I was in with my GP and I said to him about a change of tablets.

(Peter, 68 years)

...very helpful, and the fact last time I was with the GP he changed my medication.

(John, 82 years)

When asked, participants stated that they would be happy to participate in a similar project or full 12month trial, and some felt it would be something they would like to have as part of their care package on an ongoing basis.

I'd be quite happy to do it if it was of benefit to him (GP) or to myself.

(Robert, 62 years)

Certainly, I would recommend it, anybody of my age I would recommend to have a monitor. 
In general patients found the intervention acceptable and easy to use. However, this intervention may not suit all patients post stroke. It is important that they understand that they can contact their GP for reassurance if their readings are high and that they have clear information on how to contact the research nurse for further information or to answer any questions. Results from this qualitative work have demonstrated key outcome measures of recruitment, retention, adherence to the protocol and general acceptability of the intervention.

\section{GP views}

Individual semi-structured interviews were carried out with the four participating GPs. They found the intervention acceptable, and easy to incorporate in to their practice activity. They didn't identify an increased workload as the general consensus was that this was clinical activity that needed to be done anyway.

...because you know it's something that has to be done, it would have a negligible impact really. We set aside time for checking results and acting on them anyway.

(GP, small urban practice)

Getting regular updates and information about somebody's blood pressure control, has the potential to, I've no doubt, save consultations on the one hand, and certainly save in terms of morbidity and mortality associated with potential events that you're preventing.

(GP, small rural practice)

There was a general recommendation for results to be delivered via their standard practice electronic results delivery system Healthlink (the national hospital and labs results system) rather than by email, which would ensure that the results were reviewed and actioned during practice hours and in line with standard practice protocols for reviewing results.

It would be preferable if it could be linked directly to the EMR the electronic patient medical record.

(GP, small rural practice)

If it was connected with the GP software that would be more user friendly.

(GP, large mixed urban and rural practice)

They had no problems making treatment decisions based on the results of the home BP readings and commented that it encouraged their patients to become more involved in their care.

...people are living much longer lives, they are living with multiple chronic diseases, so I guess making patients not just passive receivers of a service or just passive recipients of care, but rather, making them 
sort of active agents in their own health and wellness, I think is really important. I think technology and this example of technology is a great enabler in terms of that.

(GP, small rural practice)

From what I can see, yeah, delighted with it and delighted to be involved in their care.

(GP, large urban practice)

They all indicated a willingness to continue with the intervention for a full trial or as part of a patient's ongoing care plan and felt that patients were happy with the system.

I was amazed about some of my patients, I was worried that some of them might not be able to, you know, or might have reservations about partaking, but I mean they all seem to like it.

(GP, small urban practice)

\section{Discussion}

Summary

We have demonstrated that blood pressure self-monitoring using the TASMIN5S system of digital feedback, is both feasible and acceptable with stroke patients and GPs. This study reports on the use of a GP supervised self-monitoring system for patients, with agreed treatment escalation following the feedback of results via a digital platform. GPs were willing to take part in the study with eight out of ten practices consenting to take part when approached initially. There was no reported effect on practice time or increase in GP workload. Of the patients randomised, 93\% (14/15) completed the study and there were no adverse events. A significant reduction in systolic BP and diastolic BP was seen in the intervention group at 12 weeks.

Comparison with existing literature

The current ESC/ESH (European Society of Cardiology/ European Society of Hypertension) guidelines recommend a target Systolic BP of $120-130 \mathrm{mmHg}(11)$ for patients post stroke. There is strong evidence that lowering blood pressure to optimal targets has a positive effect on the prevention of further stroke $(31,32)$. Our study aimed for a systolic BP target of less than $125 \mathrm{~mm}$ (European $\mathrm{Hg}$ on home readings. Many participants reached this target BP without any adverse effects.

There is an increasing body of literature examining the potential beneficial effects of blood pressure selfmonitoring particularly when associated with co-interventions (33-36). Although improvements in BP control were often small in these studies, this may still have an important clinical effect in reducing vascular complications in hypertensive patients. Self-monitoring empowers patients, is cost effective and is well tolerated (37). It has been shown to be superior than office BP monitoring in predicting end organ damage (38). Combining blood pressure self-monitoring with co-interventions such as tele-monitoring 
and tailored support for patients may increase these benefits (34). Designing co-interventions which involve text messaging systems as used in our study, were found to be convenient, accessible and easy to manage. None of our participants chose the option of web-based systems, all of them citing concern re internet coverage. Many were living in rural areas and were more confident in sending and receiving text messages.

Results from this study are similar to other recent studies showing that high-risk individuals with significant cardiovascular comorbidities are able to self-monitor and titrate medication adjustments in conjunction with their GP (12). Electronic messaging is increasingly being used as a support mechanism in person-centred goal achievement and has been found to be acceptable by patients in the secondary prevention of stroke (39) which is also clear in the qualitative data and the completion rate seen in our study.

\section{Strengths and Limitations}

The strengths of this study include the combination of quantitative outcomes and qualitative data, to develop an in-depth understanding of the feasibility and acceptability of this intervention. It demonstrates the implementation of the intervention in varied contexts, both before and during the Covid-19 pandemic.

Limitations to this study include the small number of general practices participating in the study due to the effects of the Covid-19 pandemic, resulting in fewer patients recruited to the study than originally planned. The original aim was to recruit 50 patients to this feasibility study which is consistent with recommendations in the literature and other similar studies in this field (40-42). However, despite smaller numbers than planned, this research provides key feasibility findings which will help in the design and development of a definitive randomised controlled trial. It should also be noted that participants were only asked to self-monitor for three months, so the findings regarding levels of engagement and acceptability may not apply when people are asked to self-monitor for a longer period of time. For example, it's not known how the anxiety brought about in one participant by ongoing high readings might impact on her longer-term engagement with the intervention.

Implications for research and practice

The current study has demonstrated successful recruitment and retention of patients in an intervention using blood pressure self-monitoring in patients post stroke. The intervention was acceptable to GPs without increasing their workload and improvements were identified that would allow results to be incorporated more easily in to their current practice-based systems. Knowledge gathered on the number of eligible patients post stroke/TIA in each practice, invitation response rate, exclusion rate and retention rate will help to inform power calculations and determine how many practices would need to be recruited for a definitive trial. This information will ensure a high quality, well-designed future trial essential for assessing the efficacy of the intervention. 
This study demonstrated a preference in the use of text messaging for delivery and feedback of results, especially among older patients and those where broadband coverage may be an issue such as in rural regions. Recruitment of a more diverse group of patients was improved following a phone call from the GP offering support and encouragement to the patient. The intervention was easily managed by patients with aphasia or lower literacy levels with aphasia friendly literature provided to all participants, and where necessary, support from a carer or family member was encouraged. System improvements for the transfer of results to GPs were identified. Patient and Public Involvement (PPI) informed the various iterations of the research design process, providing an intervention that was accessible and acceptable to both patients and clinicians.

Although the initial study protocol did not plan to recruit and assess patients in their homes, the advent of Covid-19 challenged the researchers to make changes to the original protocol in order to allow the study continue. The results of this were striking with an equal number of patients consenting to participate virtually as did face-to-face. There was no difference in the completion rates for the virtual versus face-toface groups. This provides important information for future research where virtual recruitment should be considered as an option for recruitment to future trials.

\section{Conclusion}

Blood pressure self-monitoring, using an integrated feedback system, in high-risk cardiovascular patients post stroke or TIA is both feasible and acceptable. The use of a pre-agreed three-step medication titration plan was easily implemented, increased patient involvement in their care, improved BP control and had no adverse effects. The results of this feasibility study provide valuable information for the development of a definitive RCT to determine the potential effectiveness of this intervention in patients post stroke or TIA.

\section{Abbreviations}

TIA: Transient Ischaemic Attack; IRL: Ireland; UK: United Kingdom; BP: Blood Pressure; GP: General Practitioner; RCT: Randomised Controlled Trial; BMI: Body Mass Index; EMRs: Electronic Medical Records; PCRS: Primary Care Reimbursement Service; SD: Standard Deviation; ESC/ESH European Society of Cardiology/ European Society of Hypertension; PPI: Patient and Public Involvement.

\section{Declarations}

\section{Ethics approval and consent to participate}

Research ethics approval was received from University Hospital Limerick Research Ethics Committee (Ref: 077/19). All participants provided written informed consent before data collection began. Pseudonyms are used in the reporting of qualitative data.

\section{Consent for publication}


All authors have consented to the publication of this research as presented in this manuscript.

\section{Availability of data and materials}

Data from this clinical trial will be available upon reasonable request to the corresponding author.

\section{Competing interests}

RJM has received BP monitors for research from Omron and is working with them on the development of a telemonitoring system. There are no other competing interests.

\section{Funding}

The intervention development and UK trial was supported by the Stroke Association, UK. The Irish trial was supported by the Health Research Board in Ireland. RD has received anEducation and Health Science (Ireland) scholarship.

\section{Authors' contributions}

RJM conceptualised the project and developed the research proposal and study design. RD, PH and LGG developed the study design for Ireland. CR designed and developed the software and worked with RD in adapting the system for Ireland. RD conducted the data collection. RD, LGG, PH and AS analysed the quantitative data and $\mathrm{RD}$ an $\mathrm{RH}$ analysed the interview data. RD drafted the article. All authors contributed to the manuscript, contributed to critical revision of the manuscript and gave approval of the final version to be published. LGG is the guarantor for this work.

\section{Acknowledgements}

We would like to thank all our study participants who trialled the intervention and provided valuable information during the post study interviews. We are very grateful tothe GP practices that took part in the study and for helping with patient identification and recruitment. We appreciate their participation in the qualitative study, providing key insights which will help improve the intervention for a future trial.

\section{References}

1. Kim J, Thayabaranathan T, Donnan GA, Howard G, Howard VJ, Rothwell PM, et al. Global Stroke Statistics 2019. International Journal of Stroke. 2020;15(8):819-38.

2. Wilkins E, Wilson L, Wickramasinghe K, Bhatnagar P, Leal J, Luengo-Fernandez R, et al. European Cardiovascular Disease Statistics 2017. Brussels; 2017.

3. Luengo-Fernandez R, Violato M, Candio P, Leal J. Economic burden of stroke across Europe: A population-based cost analysis. European stroke journal. 2020;5(1):17-25.

4. Mohan KM, Wolfe CD, Rudd AG, Heuschmann PU, Kolominsky-Rabas PL, Grieve AP. Risk and cumulative risk of stroke recurrence: a systematic review and meta-analysis. Stroke. 
2011;42(5):1489-94.

5. Norrving B, Barrick J, Davalos A, Dichgans M, Cordonnier C, Guekht A, et al. Action Plan for Stroke in Europe 2018-2030. Eur Stroke J. 2018;3(4):309-36.

6. Law MR, Morris JK, Wald NJ. Use of blood pressure lowering drugs in the prevention of cardiovascular disease: meta-analysis of 147 randomised trials in the context of expectations from prospective epidemiological studies. BMJ. 2009;338:b1665.

7. Webb A, Heldner M, Aguiar de Sousa D, Sandset E, Randall G, Bejot Y, et al. Availability of secondary prevention services after stroke in Europe: An ESO/SAFE survey of national scientific societies and stroke experts. European Stroke Journal. 2019;4(2):110-8.

8. Doogue R, McCann D, Fitzgerald N, Murphy AW, Glynn LG, Hayes P. Blood pressure control in patients with a previous stroke/transient ischaemic attack in primary care in Ireland: a cross sectional study. BMC Family Practice. 2020;21(1):139.

9. Hameed MA, Dasgupta I. Medication adherence and treatment-resistant hypertension: a review. Drugs Context. 2019;8:212560.

10. Ogedegbe G. Barriers to optimal hypertension control. The Journal of Clinical Hypertension. 2008;10(8):644-6.

11. Williams B, Mancia G, Spiering W, Agabiti Rosei E, Azizi M, Burnier M, et al. 2018 ESC/ESH Guidelines for the management of arterial hypertension. European Heart Journal. 2018;39(33):3021-104.

12. McManus RJ, Mant J, Haque MS, Bray EP, Bryan S, Greenfield SM, et al. Effect of self-monitoring and medication self-titration on systolic blood pressure in hypertensive patients at high risk of cardiovascular disease: the TASMIN-SR randomized clinical trial. JAMA. 2014;312(8):799-808.

13. McKinstry B, Hanley J, Wild S, Pagliari C, Paterson M, Lewis S, et al. Telemonitoring based service redesign for the management of uncontrolled hypertension: multicentre randomised controlled trial. BMJ. 2013;346:f3030.

14. Eldridge SM, Chan CL, Campbell MJ, Bond CM, Hopewell S, Thabane L, et al. CONSORT 2010 statement: extension to randomised pilot and feasibility trials. Pilot and Feasibility Studies. 2016;2(1):64.

15. Rai T, Morton K, Roman C, Doogue R, Rice C, Williams M, et al. Optimizing a digital intervention for managing blood pressure in stroke patients using a diverse sample: Integrating the person-based approach and patient and public involvement. Health Expectations. 2020;00:1-14.

16. Health Service Executive. Primary Care Reimbursement Service. Statistical Analysis of Claims and Payments 2018. Health Service Executive, Primary Care Reimbursement Service, Dublin; 2018.

17. Mattu GS, Heran BS, Wright JM. Overall accuracy of the BpTRU ${ }^{\mathrm{TM}}$-an automated electronic blood pressure device. Blood pressure monitoring. 2004;9(1):47-52.

18. Randomization Service for Multicenter Clinical Trials http://www.randomizer.at/demo/trials/1743/subject-generator-result. Accessed 01 December 2019. 
19. Katzman R, Brown T, Fuld P, Peck A, Schechter R, Schimmel H. Validation of a short OrientationMemory-Concentration Test of cognitive impairment. The American Journal of Psychiatry. 1983;140(6):734-9.

20. Intercollegiate Stroke Working Party. National Clinical Guideline for Stroke, 5th edition. London: Royal College of Physicians; 2016.

21. Belghazi J, El Feghali RN, Moussalem T, Rejdych M, Asmar RG. Validation of four automatic devices for self-measurement of blood pressure according to the International Protocol of the European Society of Hypertension. Vasc Health Risk Manag. 2007;3(4):389-400.

22. Kallio H, Pietila AM, Johnson M, Kangasniemi M. Systematic methodological review: developing a framework for a qualitative semi-structured interview guide. J Adv Nurs. 2016;72(12):2954-65.

23. Arain M, Campbell MJ, Cooper CL, Lancaster GA. What is a pilot or feasibility study? A review of current practice and editorial policy. BMC medical research methodology. 2010;10(1):1-7.

24. IBM. IBM SPSS Statistics for Windows. Version 26 ed. Aramonk, NY: IBM Corp.; 2018. p. Aramonk, NY.

25. Ritchie J, Spencer L. Qualitative data analysis for applied policy research. Analyzing qualitative data: Routledge; 2002. p. 187-208.

26. Pope C, Ziebland S, Mays N. Qualitative research in health care: Analysing qualitative data. BMJ: British Medical Journal. 2000;320(7227):114.

27. Richards L. Handling qualitative data: A practical guide: Sage; 2020.

28. QSR International Pty Ltd. NVivo. 2020.

29. Pitney WA, Parker J. Qualitative research in physical activity and the health professions: Human Kinetics Champaign, IL; 2009.

30. O'Kelly M, Teljeur C, O’Kelly F, Ni Shúilleabháin A, O’Dowd T. Structure of General Practice in Ireland 1982 - 2015. Dublin: Department of Public Health and Primary Care, Trinity College Centre for Health Sciences; 2016.

31. Katsanos AH, Filippatou A, Manios E, Deftereos S, Parissis J, Frogoudaki A, et al. Blood Pressure Reduction and Secondary Stroke Prevention: A Systematic Review and Metaregression Analysis of Randomized Clinical Trials. Hypertension. 2017;69(1):171-9.

32. Xie X, Atkins E, Lv J, Bennett A, Neal B, Ninomiya T, et al. Effects of intensive blood pressure lowering on cardiovascular and renal outcomes: updated systematic review and meta-analysis. Lancet. 2016;387(10017):435-43.

33. Uhlig K, Balk EM, Patel K, Ip S, Kitsios GD, Obadan NO, et al. Self-measured blood pressure monitoring: comparative effectiveness. U.S. Department of Health and Human Services; 2012.

34. Tucker KL, Sheppard JP, Stevens R, Bosworth HB, Bove A, Bray EP, et al. Self-monitoring of blood pressure in hypertension: A systematic review and individual patient data meta-analysis. PLoS Med. 2017;14(9):e1002389. 
35. Cappuccio FP, Kerry SM, Forbes L, Donald A. Blood pressure control by home monitoring: metaanalysis of randomised trials. BMJ. 2004;329(7458):145.

36. Bray EP, Holder R, Mant J, McManus RJ. Does self-monitoring reduce blood pressure? Meta-analysis with meta-regression of randomized controlled trials. Annals of Medicine. 2010;42(5):371-86.

37. McManus RJ, Mant J, Roalfe A, Oakes RA, Bryan S, Pattison HM, et al. Targets and self monitoring in hypertension: randomised controlled trial and cost effectiveness analysis. BMJ (clinical research ed). 2005;331(7515):493.

38. Bobrie G, Chatellier G, Genes N, Clerson P, Vaur L, Vaisse B, et al. Cardiovascular prognosis of "masked hypertension" detected by blood pressure self-measurement in elderly treated hypertensive patients. JAMA. 2004;291(11):1342-9.

39. Cadilhac DA, Andrew NE, Busingye D, Cameron J, Thrift AG, Purvis T, et al. Pilot randomised clinical trial of an eHealth, self-management support intervention (iVERVE) for stroke: feasibility assessment in survivors 12-24 months post-event. Pilot and Feasibility Studies. 2020;6(1):172.

40. Whitehead AL, Julious SA, Cooper CL, Campbell MJ. Estimating the sample size for a pilot randomised trial to minimise the overall trial sample size for the external pilot and main trial for a continuous outcome variable. Statistical Methods in Medical Research. 2015;25(3):1057-73.

41. Hilari K, Behn N, Marshall J, Simpson A, Thomas S, Northcott S, et al. Adjustment with aphasia after stroke: study protocol for a pilot feasibility randomised controlled trial for SUpporting wellbeing through PEeR Befriending (SUPERB). Pilot and Feasibility Studies. 2019;5(1):14.

42. Thabane L, Ma J, Chu R, Cheng J, Ismaila A, Rios LP, et al. A tutorial on pilot studies: the what, why and how. BMC Medical Research Methodology. 2010;10(1):1.

\section{Figures}




\begin{tabular}{|c|c|c|}
\hline $\begin{array}{l}\text { Systolic } \\
\mathrm{BP}(\mathrm{mmHg})\end{array}$ & $\begin{array}{l}\text { Diastolic } \\
\text { BP }(\mathrm{mmHg})\end{array}$ & Category \\
\hline$\geq 181$ & $\geq 101$ & $\begin{array}{l}\text { Red } \\
\text { Participant is advised to take another reading after two minutes } \\
\text { and if BP is still red to contact the Practice. }\end{array}$ \\
\hline $135-180$ & N/A & $\begin{array}{l}\text { Amber } \\
\text { Participant is advised That a medication change is recommended } \\
\text { and that they can contact their GP to confirm they are ready to } \\
\text { receive the next medication in their plan. }\end{array}$ \\
\hline $125-134$ & N/A & $\begin{array}{l}\text { If the systolic average of } 12 \text { readings is } 125-134 \text { the participant } \\
\text { is asked to take another } 12 \text { readings. } \\
\text { After } 24 \text { readings, if the systolic average is } 125 \mathrm{mmHg} \text { or above, } \\
\text { the participant receives automated feedback that their doctor } \\
\text { will take a decision for the next medication change. }\end{array}$ \\
\hline $101-124$ & N/A & $\begin{array}{l}\text { Green } \\
\text { If the systolic average of } 12 \text { readings is } 101-124 \text { the participant's } \\
\text { BP is well controlled. } \\
\text { They are congratulated and told to continue with their current } \\
\text { medication and monitor again next month. }\end{array}$ \\
\hline$\leq 100$ & $\mathrm{~N} / \mathrm{A}$ & Blue \\
\hline
\end{tabular}

\section{Figure 1}

Text messages using traffic light system 


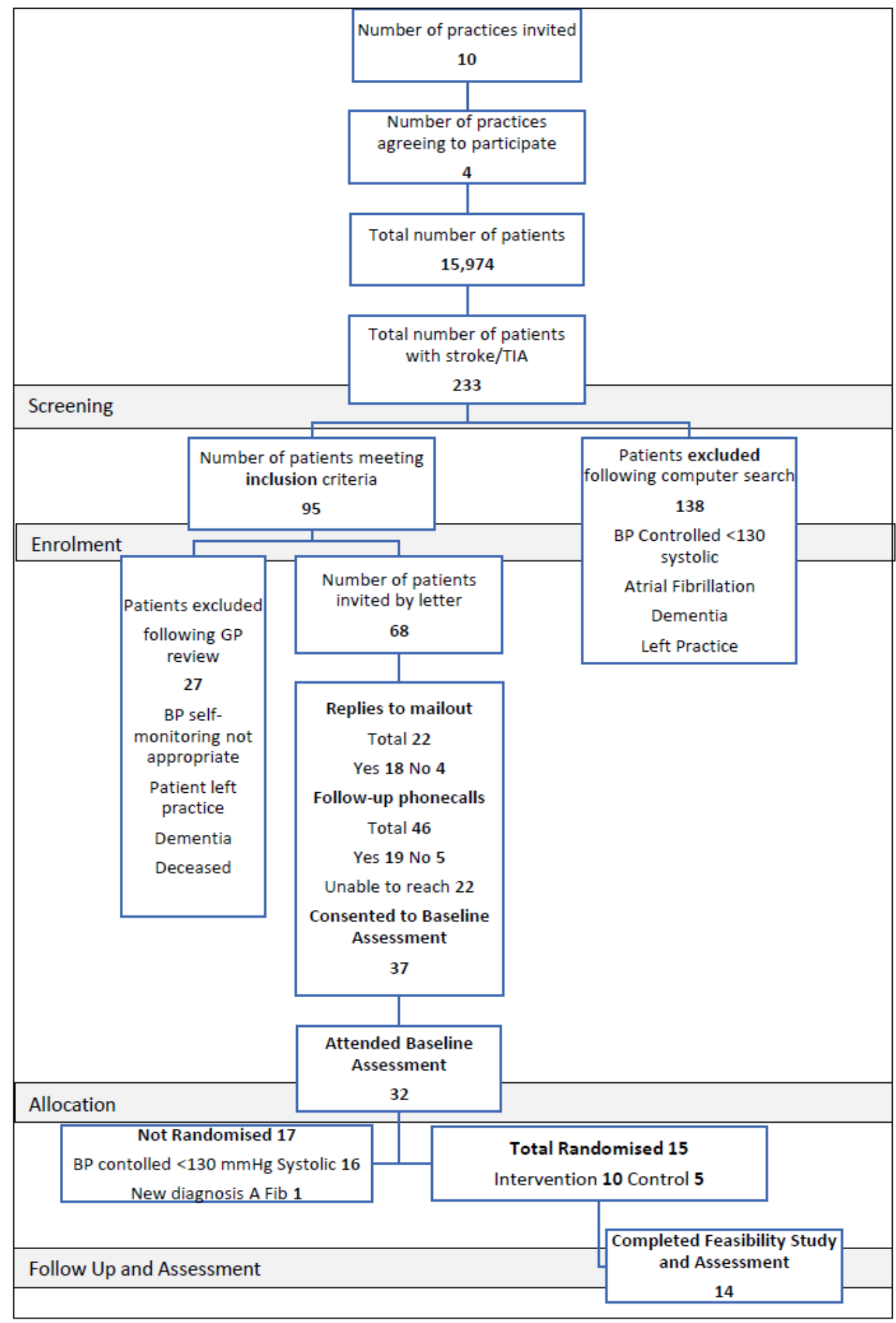

\section{Figure 2}

CONSORT diagram showing flow-through study

\section{Supplementary Files}

This is a list of supplementary files associated with this preprint. Click to download. 
- Appendix.docx

- consortchecklistBMCPilotandFeasibilitystudies.doc 